\title{
Preliminary Study of the Thai Version of the Scale for the Assessment of Negative Symptoms (SANS-Thai)
}

\author{
Thammanard Charernboon ${ }^{1,2,3}$ \\ ${ }^{1}$ Department of Psychiatry, Faculty of Medicine, Thammasat University, Pathumthani, Thailand \\ ${ }^{2}$ Department of Clinical Epidemiology, Faculty of Medicine, Thammasat University, Pathumthani, Thailand \\ ${ }^{3}$ Center of Excellence in Applied Epidemiology, Thammasat University, Pathumthani, Thailand \\ Correspondence: Thammanard Charernboon, MD, PhD, Department of Psychiatry, Faculty of Medicine, \\ Thammasat University, Phahon Yothin Road, Pathumthani 12120, Thailand. Tel: 66-29-26-9204. E-mail: \\ dr.thammanard@gmail.com
}

Received: March 12, 2019 Accepted: April 14, 2019 Online Published: April 24, 2019

doi:10.5539/gjhs.v11n6p19 URL: https://doi.org/10.5539/gjhs.v11n6p19

\begin{abstract}
Objectives: The study aimed to evaluate content validity, convergent validity, internal consistency and test-retest reliability of the Thai version of the Scale for the Assessment of Negative Symptoms (SANS-Thai).

Methods: The content validity of the SANS-Thai was assessed using four experts. The average-content validity index and item level content validity index were analyzed. The SANS-Thai and the Thai versions of the Addenbrooke's Cognitive Examination (ACE) were administered to 40 people with schizophrenia to examine convergent validity and internal consistency. Twenty participants took the second SANS-Thai assessment within four weeks to evaluate test-retest reliability.

Results: The results demonstrated that the SANS-Thai has excellent content validity with the average-content validity index of 0.94 . The majority of the item level content validity index range from 0.75 to 1 . The global and total SANS-Thai score moderately correlated with the ACE with the correlation coefficient of $-0.48(\mathrm{p}=0.002)$ and $-0.49(\mathrm{p}=0.001)$, respectively. Internal consistency by the Cronbach alpha coefficient was 0.95 . Test-retest reliabilities by intraclass correlation were $0.91(\mathrm{p}<0.001)$ for global SANS-Thai and $0.9(\mathrm{p}<0.001)$ for total SANS-Thai. The Bland-Altman plot demonstrated that only $5 \%$ of the participants fell outside the limits of agreement for both global SANS and total SANS scores.
\end{abstract}

Conclusion: The SANS-Thai appears to be a valid and reliable measure of negative symptoms in schizophrenia and could be useful for patient care and research studies.

Keywords: negative symptoms, psychopathology, rating scale, schizophrenia

\section{Introduction}

Negative symptoms have long been noted as a common feature of schizophrenia and as such remains one of the diagnostic criteria of schizophrenia in the Diagnostic and Statistical Manual of Mental Disorders (DSM)-IV and DSM-5 (American Psychiatric Association, 2013). Patients who exhibit substantial negative symptoms have particularly poor functional outcome and it has been proposed as a separate domain with distinctive pathological and therapeutic implications (Kirkpatrick, Fenton, Carpenter, \& Marder, 2006). Therefore, the treatment of negative symptoms is an essential aspect in schizophrenia management, and negative symptoms need to be accurate and reliably assessed.

The Scale for the Assessment of Negative Symptoms (SANS) is one of the most widely used negative symptom scales in both clinical trials and practice (Andreasen, 1982, 1984). It was developed specifically for the purpose of assessing negative symptoms in schizophrenia and has been translated into many languages such as (Kulhara, Kota, \& Joseph, 1986; Phillips et al., 1991). However, a Thai version has not been available before now. Moreover, accurate translation and assessment of the validity and reliability of the instruments must take precedence when developing a measurement in different languages and cultures. Hence, the objectives of the present study were to evaluate the validity and reliability of the SANS-Thai. 


\section{Methods}

\subsection{Participants and Study Procedure}

The study consisted of two parts: 1) translation and contents validity and 2) validity and reliability in people with schizophrenia.

A translation process comprised of two psychiatric experts, familiar with the SANS, translated the English version of SANS into Thai. This was followed by a professional translator translating the first drafts into English. The forward and back translated versions were reviewed by taking into account the assessment concepts, language usage and complexities, as well as cultural appropriateness. All definitions of symptoms were unchanged from the original English version; only minor adaptations of examples of the symptoms were made.

After the translation process, the author invited four psychiatrists to participate in the content validity study of the Thai-SANS. Each item was rated on a 4-point scale $(0=$ not relevant; $3=$ highly relevant $)$. A content validity index was calculated both at the item level (item-CVI) and average content validity index (average-CVI). The item-CVI was calculated as the number of experts giving a score of 2 or 3 divided ty the total number of experts. The average-CVI was the average proportion of all items rated as 2 or 3 across the four experts.

The second part of the study was to assess convergent validity, internal consistency, and test-retest reliability in people with schizophrenia. Participants included were 40 clinically stable schizophrenia patients from the psychiatric clinic at Thammasat University Hospital, Thailand during 1 May 2016 to 31 December 2016. All participants were diagnosed using DSM-5 criteria of schizophrenia by certified psychiatrists.

Demographic data on the patients included gender, age and level of education. One psychiatrist interviewed them and rated the SANS-Thai. Research assistants, who were blinded to the SANS-Thai score, then evaluated general neurocognition using the Thai version of the Addenbrooke's Cognitive Examination (ACE) (Charernboon, Jaisin, \& Lerthattasilp, 2016). To evaluate test-retest reliability, 20 participants completed the second SANS assessment within 2-4 weeks.

The present study was part of our larger study and approved by the Human Ethics Committee of Faculty of Medicine, Thammasat University (protocol number: MTU-EC-ES-6-043/59). Some of the participants have contributed the SAPS and SANS data to our previous report (Charernboon \& Patumanond, 2017).

\subsection{Measures}

\section{The Thai version of the Scale for the Assessment of Negative Symptoms (SANS-Thai)}

The SANS-Thai consists of 20 items and is divided into five domains, which are affective flattening (7 items), alogia (4 items), avolition-apathy (3 items), anhedonia-asociality (4 items) and attention (2 items)(Andreasen, 1984). Each item rating was on a six-point scale ranging from 0 to 5 . Each domain also included a global rating score which is the summarized score of the symptoms in that domain. The 'total SANS score' can be calculated by adding the score of all 20 items, ranging from 0 to 100 . The 'global SANS score' is calculated by summarizing the score of each global rating scale, ranging from 0 to 25 . A higher score indicated higher severity of the symptoms.

\section{The Thai version of the Addenbrooke's Cognitive Examination III (ACE)}

The Addenbrooke's Cognitive Examination III (ACE) is an assessment of general neurocognition (Charernboon et al., 2016; Hsieh, Schubert, Hoon, Mioshi, \& Hodges, 2013). Scores range from 0 to 100, with higher scores indicating better neurocognitive function. The ACE was used to evaluate convergent validity with SANS-Thai. Studies have found that negative symptoms and cognitive deficits share some features and usually are correlated in their severity in schizophrenia (Harvey, Koren, Reichenberg, \& Bowie, 2006).

\subsection{Statistical Analysis}

Pearson correlations were used to measure the convergent validity between the global SANS-Thai and total SANS-Thai compared to the ACE. Internal consistency was assessed with the Cronbach alpha. Test-retest reliability was evaluated with the intraclass correlation coefficient and a Bland-Altman plot. The Bland-Altman plot demonstrated the average of the first and second assessment against all differences between first and second assessment (test-retest score) and estimated limits of agreement. A p-value of $<0.05$ indicated statistical significance. All analyzes were performed using STATA version 14. 


\section{Results}

The participants consisted of 40 schizophrenia patients: 21 females and 19 males. Their mean age was 36.9 (SD 12.8) years and education level was 13.1 (SD 3.6) years.

\subsection{Content Validity}

The results demonstrated that SANS-Thai has excellent content validity with the average-CVI of 0.94 . The majority of the item-CVIs ranged from 0.75 to 1 . Only one item, inattentiveness during mental status testing, had an item-CVI of 0.5 (Table 1).

Table 1 . Content validity

\begin{tabular}{|c|c|c|c|c|c|}
\hline Items & Expert1 & Expert2 & Expert3 & Expert4 & Item CVI \\
\hline \multicolumn{6}{|l|}{ Affective Flattening or Blunting } \\
\hline 1. Unchanged facial expression & l & l & l & l & 1 \\
\hline 2. Decreased spontaneous movements & l & / & l & l & 1 \\
\hline 3. Paucity of expressive gestures & l & I & l & l & 1 \\
\hline 4. Poor eye contact & l & l & l & l & 1 \\
\hline 5. Affective nonresponsiveness & I & l & l & - & 0.75 \\
\hline 6. Lack of vocal inflections & l & l & l & l & 1 \\
\hline 7. Inappropriate affect & l & - & l & l & 0.75 \\
\hline
\end{tabular}

\section{Alogia}

1. Poverty of speech

2. Poverty of content of speech

3. Blocking

4. Increased latency of response

$\begin{array}{lllll}1 & 1 & 1 & 1 & 1 \\ 1 & 1 & 1 & 1 & 1 \\ 1 & 1 & 1 & 1 & 1 \\ 1 & 1 & 1 & 1 & 1\end{array}$

\section{Avolition-Apathy}

1. Grooming and hygiene

2. Impersistence at work or school

3. Physical Anergia

$\begin{array}{ll}1 & 1 \\ 1 & 1 \\ 1 & 1\end{array}$

\section{Anhedonia-Asociality}

1. Recreational interests and activities

2. Sexual activity

3. Ability to feel intimacy and closeness

4. Relationships with friends and peers

1
1
1
1

$\begin{array}{lll}/ & / & 1 \\ / & / & 1 \\ / & / & 1\end{array}$

\section{Attention}

\begin{tabular}{llllll} 
1. Social inattentiveness & $/$ & $/$ & $/$ & - & 0.75 \\
2. Inattentiveness during mental status testing & $/$ & - & - & $/$ & 0.5 \\
\hline Proportion relevant & 1 & 0.9 & 0.95 & 0.9 & 0.94 \\
\hline
\end{tabular}

/: relevant (rating 2 or 3), -: not relevant (rating 0 or 1 ). 


\subsection{Convergent Validity}

The correlation between the ACE and global SANS-Thai score was $-0.48(\mathrm{p}=0.002)$; the ACE and total SANS-Thai score was $-0.49(\mathrm{p}=0.001)$.

\subsection{Reliability}

The SANS-Thai had a good internal consistency with the Cronbach's alpha of 0.95 .

The test-retest reliability, as measured by intraclass correlation, was $0.91(p<0.001)$ for the global SANS-Thai score and $0.9(\mathrm{p}<0.001)$ for the total SANS-Thai score. As an additional test between test and retest, Bland-Altman plots were constructed as shown in Figures 1 and 2. For the global SANS-Thai score, the mean difference was 1.65 , and the $95 \%$ limits of agreement ranged -2.7 to 6.0 points. Only 1 out of 20 participants (5\%) fell outside the limits of agreement. Regarding the total SANS-Thai score, the mean difference was 5.1, and 95\% limits of agreement was -12.9 to 23.1 points. One out of 20 participants (5\%) fell outside the limits of agreement.

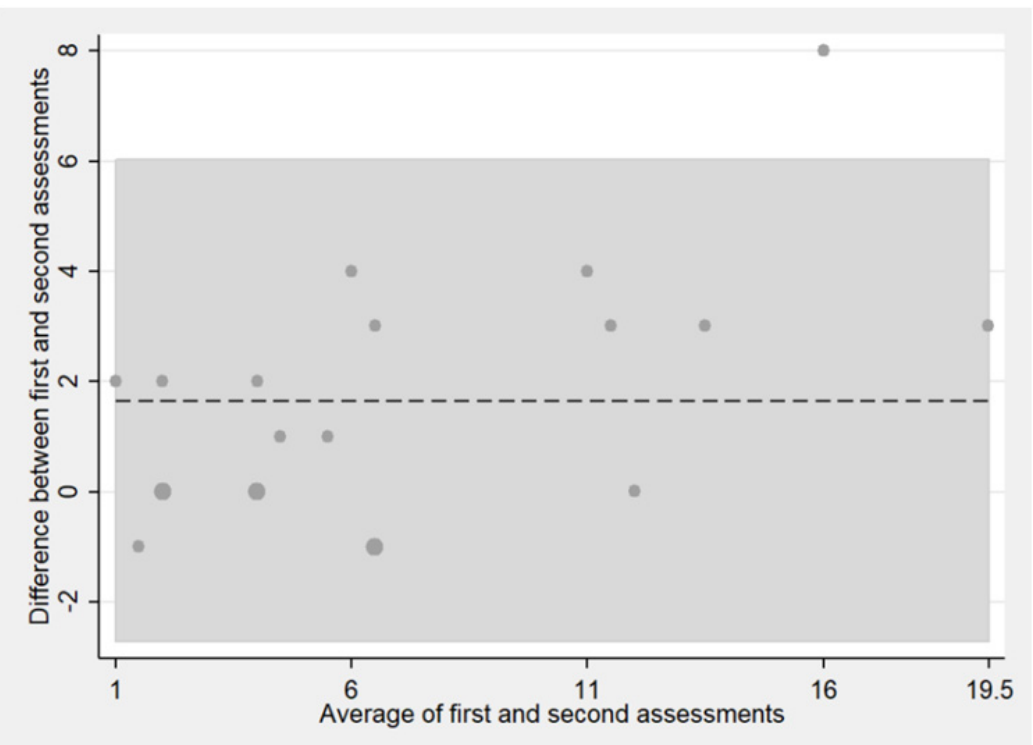

Figure 1. Bland-Altman plot of the stability of the global SANS-Thai score

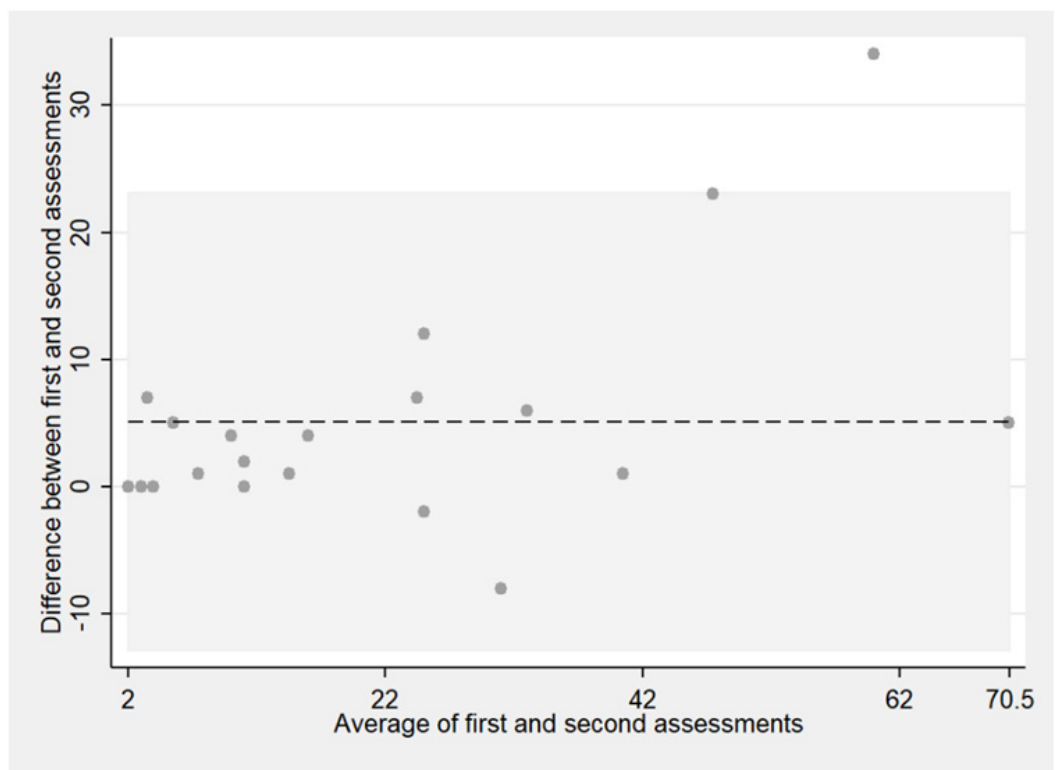

Figure 2. Bland-Altman plot of the stability of the total SANS-Thai score 


\section{Discussion}

In general, the study demonstrated that the Thai version of the SANS is valid and reliable. It was confirmed to have an excellent content validity with excellent average-CVI. The only low item-CVI score was 'inattentiveness during mental status testing'. Two experts suggested that the attention test should be considered as a cognitive function more than negative symptoms; this is the typical and generally known criticism of the SANS (Daniel, 2013).

As expected, the results showed a moderate correlation between the ACE and global SANS-Thai and total SANS-Thai scores. Multiple previous cross-sectional studies have illustrated that the severity of negative symptoms and cognitive symptoms are usually correlated. These correlations are generally moderate with the average correlation of 0.3 (Harvey et al., 2006).

The Cronbach's coefficient alphas indicated that the SANS-Thai had a high internal consistency (0.95), comparable to the original study by Andreasen which had an overall internal consistency of 0.89 (Andreasen, 1982). A multisite study of the reliability of the SANS by Mueser also demonstrated an internal consistency of the total score of 0.89 (Mueser, Sayers, Schooler, Mance, \& Haas, 1994).

The SANS-Thai had excellent test-retest reliability with intraclass correlations of more than 0.9 for both the global and total score. The Bland-Altman plot showed that $95 \%$ of the differences between test and retest lie within the limits of agreement for both the global and total scores. The test-retest reliability is quite similar to the Chinese versions of the SANS which had an intraclass correlation for the total score of 0.88 and global score of 0.82 (Phillips et al., 1991). The test-retest reliability of the original English versions of the SANS was never reported.

The main limitation was that the convergent validity with other negative symptom instruments and inter-rater reliability were not evaluated in this study. Further research should examine these psychometric properties.

\section{Conclusion}

The Thai version of the SANS provides a comprehensive measurement of negative symptoms of schizophrenia. It is valid and reliable and could be a useful tool for the research and clinical practice.

\section{Acknowledgments}

The author thanks for Keerati Pattanaseri, MD, MSc, Tiraya Lerhattasilp, MD, Winitra Nuallaong, MD, MSc and Veevarin Charoenporn, MD for the translation and development of the SANS-Thai and Piyanud Chompookard, $\mathrm{BA}$, for help in collecting the data. The author also would like to thank Prof. Nancy C Andreasen, MD, PhD for giving permission to translate the SANS.

\section{Funding}

There was no fund for this study.

\section{Competing Interests Statement}

The authors declare that there are no competing or potential conflicts of interest.

\section{References}

American Psychiatric Association. (2013). Diagnostic and Statistical Manual of Mental Disorders. Washington DC: American Psychiatric Publishing. https://doi.org/10.1176/appi.books.9780890425596

Andreasen, N. C. (1982). Negative symptoms in schizophrenia. Definition and reliability. Arch Gen Psychiatry, 39(7), 784-788. https://doi.org/10.1001/archpsyc.1982.04290070020005

Andreasen, N. C. (1984). Scale for the Assessment of Negative Symptoms (SANS). Department of Psychiatry, College of Medicine, The University of Iowa.

Charernboon, T., Jaisin, K., \& Lerthattasilp, T. (2016). The Thai Version of the Addenbrooke's Cognitive Examination III. Psychiatry Investig, 13(5), 571-573. https://doi.org/10.4306/pi.2016.13.5.571

Charernboon, T., \& Patumanond, J. (2017). Social Cognition in Schizophrenia. Ment Illn, 9(1), 7054. https://doi.org/10.4081/mi.2017.7054

Daniel, D. G. (2013). Issues in selection of instruments to measure negative symptoms. Schizophr Res, 150(2-3), 343-345. https://doi.org/10.1016/j.schres.2013.07.005

Harvey, P. D., Koren, D., Reichenberg, A., \& Bowie, C. R. (2006). Negative symptoms and cognitive deficits: what is the nature of their relationship? Schizophr Bull, 32(2), 250-258. https://doi.org/10.1093/schbul/sbj011

Hsieh, S., Schubert, S., Hoon, C., Mioshi, E., \& Hodges, J. R. (2013). Validation of the Addenbrooke's Cognitive 
Examination III in frontotemporal dementia and Alzheimer's disease. Dement Geriatr Cogn Disord, 36(3-4), 242-250. https://doi.org/10.1159/000351671

Kirkpatrick, B., Fenton, W. S., Carpenter, W. T., Jr., \& Marder, S. R. (2006). The NIMH-MATRICS consensus statement on negative symptoms. Schizophr Bull, 32(2), 214-219. https://doi.org/10.1093/schbul/sbj053

Kulhara, P., Kota, S. K., \& Joseph, S. (1986). Positive and negative subtypes of schizophrenia. A study from India. Acta Psychiatr Scand, 74(4), 353-359. https://doi.org/10.1111/j.1600-0447.1986.tb06254.x

Mueser, K. T., Sayers, S. L., Schooler, N. R., Mance, R. M., \& Haas, G. L. (1994). A multisite investigation of the reliability of the Scale for the Assessment of Negative Symptoms. Am J Psychiatry, 151(10), 1453-1462. https://doi.org/10.1176/ajp.151.10.1453

Phillips, M. R., Xiong, W., Wang, R. W., Gao, Y. H., Wang, X. Q., \& Zhang, N. P. (1991). Reliability and validity of the Chinese versions of the Scales for Assessment of Positive and Negative Symptoms. Acta Psychiatr Scand, 84(4), 364-370. https://doi.org/10.1111/j.1600-0447.1991.tb03161.x

\section{Copyrights}

Copyright for this article is retained by the author(s), with first publication rights granted to the journal.

This is an open-access article distributed under the terms and conditions of the Creative Commons Attribution license (http://creativecommons.org/licenses/by/4.0/). 